\title{
ก
}

\section{Polytechnic Institute of Coimbra}

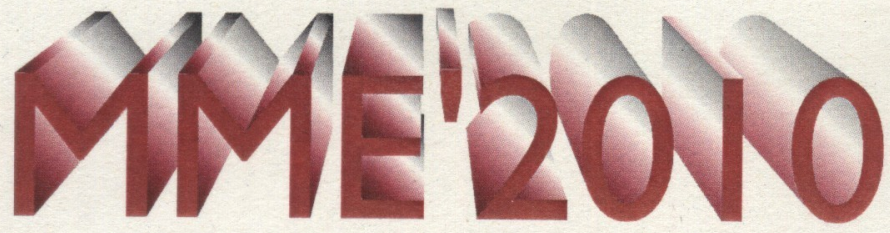

Mathemattcal Methods in Engineering International Symposfum

IPC, Coimbra. Portugal

21 - 24 October 2010 


\title{
A Statistical Approach for Tuning the Windowed Fourier Transform
}

\author{
Miguel F. M. Lima
}

Dept. of Electrical Engineering Superior School of Technology, Polytechnic Institute of Viseu, 3504-510 Viseu, Portugal

lima@mail.estv.ipv.pt

\section{J.A. Tenreiro Machado}

Dept. of Electrical Engineering, Institute of Engineering, Polytechnic Institute of Porto, 4200-072 Porto, Portugal

jtm@isep.ipp.pt

\section{Abstract}

The windowed Fourier transform is one of the most widely used time-frequency representations. In order to use this technique several parameters must be defined according to the signal analyzed. In this paper are studied the effects of the type, the shape, the length and the overlap of the windows, and new method for tuning the window parameters is presented. The study method is based on the information theory and is applied to signals captured during the movement of a robotic manipulator. The experimental results demonstrate the applicability and the effectiveness of the proposed approach. 